C员员枵
REPORT 79-19

The critical velocities of a floating ice plate subjected to in-plane forces and a moving load

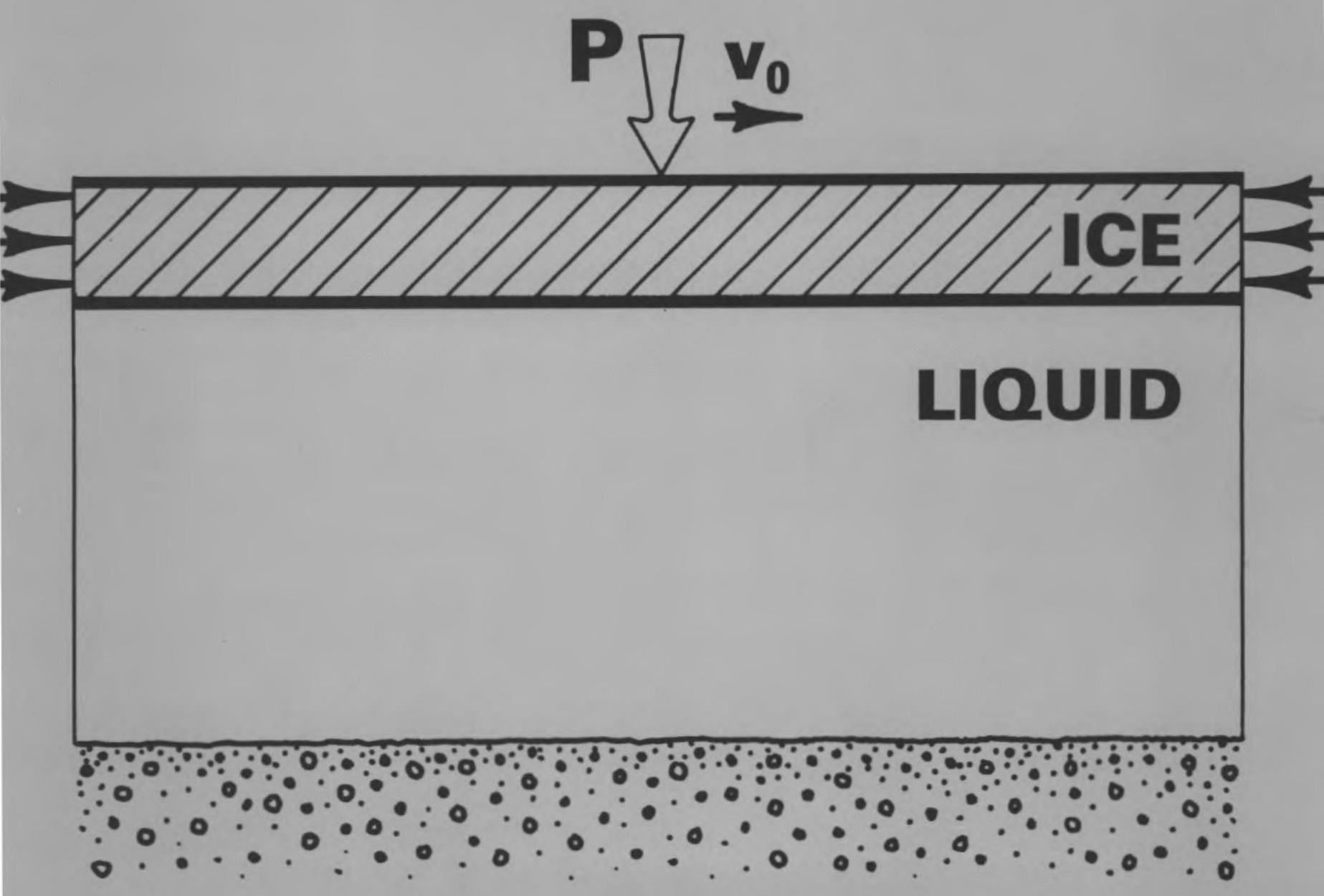




\section{CRREL Report 79-19}

The critical velocities of a floating ice plate subjected to in-plane forces and a moving load

Arnold D. Kerr

August 1979

Prepared for

DIRECTORATE OF MILITARY PROCRAMS

OFFICE, CHIEF OF ENGINEERS

By

UNITED STATES ARMY

CORPS OF ENGINEERS

COLD REGIONS RESEARCH AND ENGINEERING LABORATORY

HANOVER, NEW HAMPSHIRE, U.S.A. 


\title{
THE CRITICAL VELOCITIES OF A FLOATING ICE PLATE SUBJECTED TO IN-PLANE FORCES AND A MOVING LOAD
}

\author{
by \\ Arnold D. Kerr

\section{INTRODUCTION}

It is well known that when a vehicle is moving on a floating ice sheet there is a certain velocity, denoted in the mechanics literature as the "critical velocity" $\mathbf{v}_{\mathrm{cr}}$, at which it may break through the ice. Methods for de termining $v_{c r}$ for floating plates subjected to moving loads have been presented by Assur (1961), Kheishin $(1963,1967)$ and Nevel (1970). Kheishin and Nevel utilized the linear bending theory of plates to describe the response of the ice cover, and the equations of an ideal fluid to describe the response of the liquid base.

Recently, Kerr (1972) showed that an axial force in a beam on a Winkler base that is subjected to a moving lateral load may have a profound effect upon $v_{c r}$. Since axial in-plane forces also occur in floating ice covers, caused by constrained thermal strains, it is of interest to determine their effect upon $v_{c r}$. The purpose of the present paper is to study this phenomenon.

At first, we analyze some related problems: the magnitude of the critical in-plane compression forces for a uniform biaxial stress field, and then the propagation of free waves in the floating plate subjected to inplane stresses. This is followed by the determination of $\mathbf{v}_{\mathbf{c r}}$ for a floating plate subjected to a moving load and an in-plane force field.

\section{THE INSTABILITY OF A FLOATING INFINITE PLATE SUBJECTED TO A BIAXIAL FORCE FIELD}

It is assumed that the governing equation for the determination of $N_{\mathrm{er}}$ is

$$
D \nabla^{4} w+N \nabla^{2} w+\rho_{f} g w=0
$$

where

$$
\nabla^{4}=\frac{\partial^{4}}{\partial x^{4}}+2 \frac{\partial^{4}}{\partial x^{2} \partial y^{2}}+\frac{\partial^{4}}{\partial y^{4}}
$$

$w(x, y)$ is the perturbation from the plane state, $D$ is the flexural rigidity of the plate, and $\rho_{\mathrm{f}} \boldsymbol{g}$ is the specific weight of the liquid base. 


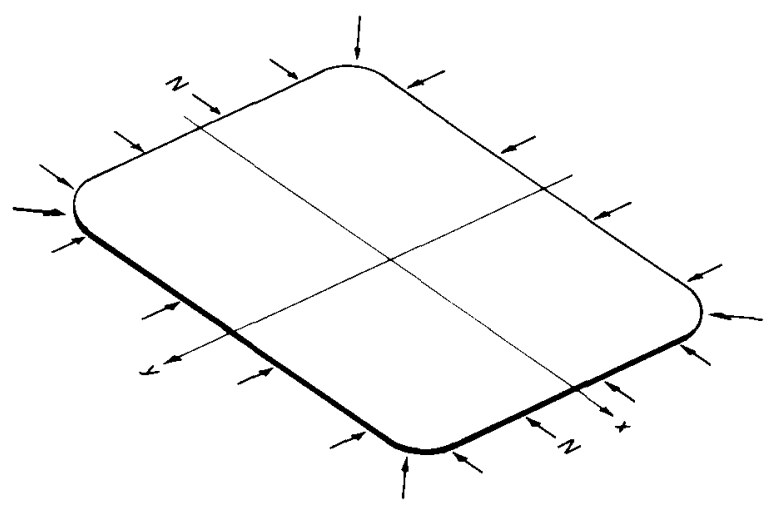

Figure 1 .

For a buckling mode of the form

$$
w(x, y)=w_{0} \sin \left(a_{1} x\right)
$$

where $w_{0}=$ const., eq 1 yields, setting $\rho_{\mathrm{f}} g / D=\kappa^{4}=1 / \ell^{4}$,

$$
\left(a_{1}^{4}-\frac{N}{D} a_{1}^{2}+\kappa^{4}\right) w_{0} \sin \left(a_{1} x\right)=0
$$

Equation 4 is satisfied for a non-zero $w$ when

$$
a_{1}^{4}-\frac{N}{D} a_{1}^{2}+k^{4}=0
$$

and thus when

$$
\frac{N}{D}=\frac{a_{1}^{4}+\kappa^{4}}{a_{1}^{2}}
$$

Since the right-hand side of eq 6 is $>0$ it follows, as anticipated, that the deformation mode of the form shown in eq 3 is only possible when $N$ is a compression field, as is shown in Figure 1. From $\partial(N / D) / \partial a_{1}=0$, it follows that $N=N_{\text {min }}$ when

$$
a_{1}=\kappa
$$

Substituting eq 7 into eq 6 , it follows that

$$
\frac{N_{\mathrm{cr}}}{D}=2 \kappa^{2}=2 \sqrt{\frac{\rho_{\mathrm{f}} g}{D}}
$$

or, rewritten,

$$
N_{\mathrm{cr}}=2 \sqrt{\rho g}{ }^{*}
$$

The corresponding wave length is

$$
\lambda_{\mathrm{b}}=\frac{2 \pi}{a_{1}}=\frac{2 \pi}{\kappa}=2 \pi \ell=2 \pi \sqrt[4]{\frac{D}{\rho_{\mathrm{f}} g}} .
$$

\section{THE PROPAGATION OF FREE WAVES}

\section{The liquid layer}

Assuming that the liquid layer (Fig. 2) responds like an ideal fluid (Kheishin 1963), and that the ratio of amplitude to wave length is very small, the resulting equations, in terms of the velocity potential $\Phi$, are:

- The same result is obtained for $w(x)=w_{0} \sin \left(a_{1} x\right) \sin \left(a_{2} y\right)$. However then, instead of eq $7, \kappa^{2}=a_{1}^{2}+a_{2}^{2}$. 


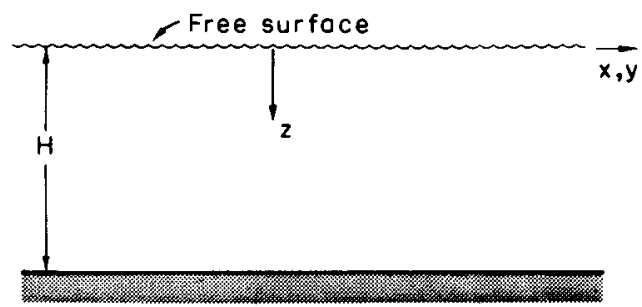

Figure 2.

$$
\begin{aligned}
& \nabla_{\mathrm{x}, \mathrm{y}, \mathrm{z}}^{2} \Phi=0 \quad \text { for } 0 \leq z \leq H \\
& \left(\frac{\partial \Phi}{\partial z}-\frac{1}{\mathrm{~g}} \frac{\partial^{2} \Phi}{\partial t^{2}}\right)_{\mathrm{z}=0}=0 \\
& \left(\frac{\partial \Phi}{\partial z}\right)_{\mathrm{z}=\mathrm{H}}=0
\end{aligned}
$$

where

$$
\nabla_{x, y, z}^{2}=\frac{\partial^{2}}{\partial x^{2}}+\frac{\partial^{2}}{\partial y^{2}}+\frac{\partial^{2}}{\partial z^{2}}
$$

Assuming

$$
\Phi(x, z, t)=A \phi(z) \cos a(x-c t)
$$

and substituting it into eq 11 , it follows that $\phi(z)$ has to satisfy

$$
\frac{d^{2} \phi}{d z^{2}}-a^{2} \phi=0 \quad \text { for } 0 \leq z \leq H
$$

The general solution of the above differential equation is

$$
\phi(z)=B_{1} \mathrm{e}^{+\alpha z}+B_{2} \mathrm{e}^{-\alpha z} .
$$

Noting boundary condition 13 , it follows that

$$
\phi(z)=2 B_{1} \mathrm{e}^{a H} \cosh a(H-z) .
$$

Substitution of eq 15 and 14 into the remaining boundary condition (12) yields

$$
2 A B_{1} \mathrm{e}^{\alpha H}\left[-\alpha \sinh \alpha(H-z)+\frac{a^{2} c^{2}}{g} \cosh \alpha(H-z)\right]_{z=0} \cos \alpha(x-c t)=0 .
$$

The above equation is satisfied, for any amplitude of $\Phi$, when

$$
c_{\mathrm{f}}^{2}=\frac{g}{a} \operatorname{tgh}(a H)
$$

Noting that $a=2 \pi / \lambda$ it follows that a solution of the form 14 will exist when the phase velocity $c$ and the wave length $\lambda$ are related by the dispersion relation:

$$
c_{\mathrm{f}}=\sqrt{\frac{g \lambda}{2 \pi} \operatorname{tgh}\left(\frac{2 \pi H}{\lambda}\right)}
$$




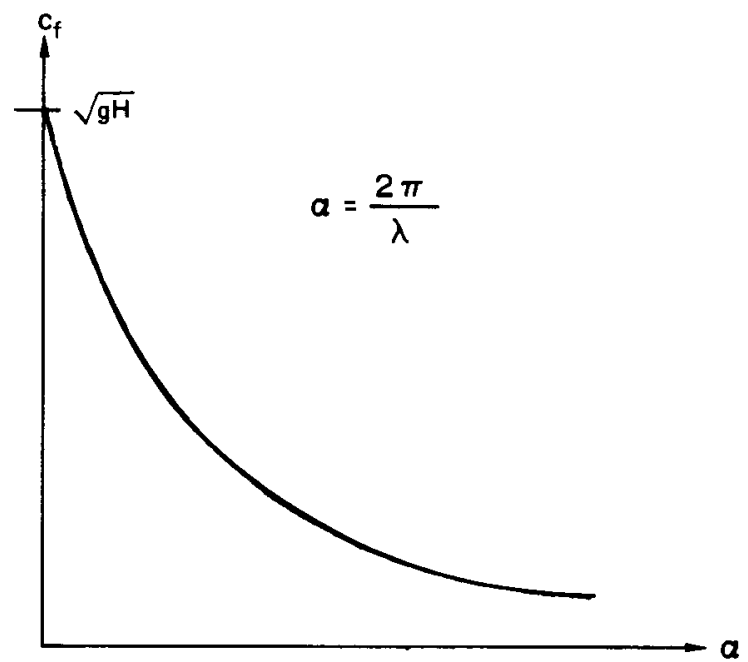

Figure 3.
Equation 16 is presented schematically in Figure 3. Thus, there exists a range of $0 \leqq c<\infty$ and to each phase velocity there corresponds a wave length $\lambda$.

It may be shown (Lamb 1945) that to the above solution there corresponds a sinusoidal free surface wave of wave length $\lambda$ which travels in the $x$ direction with the velocity $c$. For additional comments the reader is referred to the literature on free waves in liquids.

\section{The stressed plate (without base)}

Assuming that the plate responds elastically and is subjected to a uniform in-plane force field $N$, the following plate equation is used for long waves:

$$
D \nabla_{\mathbf{x}, \mathrm{y}}^{4} w+N \nabla_{\mathrm{x}, \mathrm{y}}^{2} w+\rho_{\mathrm{p}} h \frac{\partial^{2} w}{\partial t^{2}}=0
$$

Assuming a traveling wave of the form

$$
w(x, y, t)=w_{0} \sin a(x-c t)
$$

and substituting it into the above differential equation, it follows that

$$
\left[D a^{4}-N \alpha^{2}-\rho_{\mathrm{p}} h \alpha^{2} c^{2}\right] w_{0} \sin a(x-c t)=0 .
$$

Thus, a wave of the form 18 can propagate in the plate when $N<D a^{2}$ and then $c, a$ and $N$ are related by the dispersion relation

$$
c_{\mathrm{pl}}^{2}=\frac{D \alpha^{2}-N}{\rho_{\mathrm{p}} h}
$$

Writing $N=n a^{2} D$ where $n<1$, eq 19 becomes

$$
c_{\mathrm{pl}}=\alpha \sqrt{\frac{(1-n) D}{\rho_{\mathrm{p}} h}}
$$

Equation 19a is presented schematically in Figure 4.

\section{The stressed plate resting on a liquid layer}

Retaining the assumptions made in the two preceding sections, the resulting formulation is (Kheishin 1963):

$$
D \nabla_{\mathrm{x}, \mathrm{y}}^{4} W+N \nabla_{\mathrm{x}, \mathrm{y}}^{2} W+\rho_{\mathrm{p}} h \frac{\partial^{2} w}{\partial t^{2}}+\rho_{\mathrm{f}} \mathrm{g} W-\left.\rho_{\mathrm{f}} \frac{\partial \Phi}{\partial t}\right|_{\mathrm{z}=\mathrm{w}}=0
$$




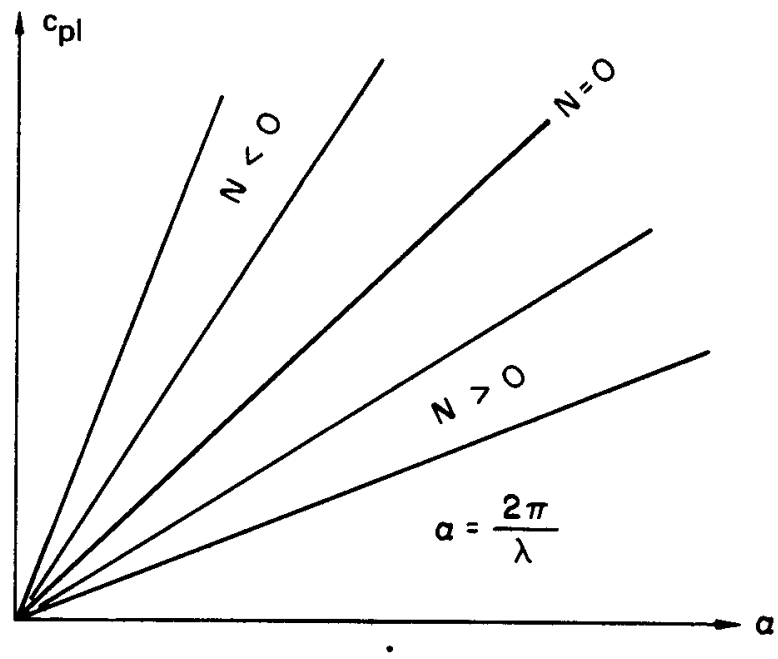

Figure 4.

$$
\begin{aligned}
& \nabla_{\mathrm{x}, \mathrm{y}, \mathrm{z}}^{2} \Phi=0 \\
& \left.\frac{\partial \Phi}{\partial z}\right|_{\mathrm{z}=\mathrm{w}}=\frac{\partial w}{\partial t} \\
& \left.\frac{\partial \Phi}{\partial z}\right|_{\mathrm{z}=\mathrm{H}}=0
\end{aligned}
$$

and regularity conditions for $w$ and $\Phi$ as $x^{2}+y^{2} \rightarrow \infty$.

It is assumed, as above, that

$$
\begin{aligned}
& w(x, y, t)=w_{0} \sin a(x-c t) \\
& \Phi(\mathrm{x}, z, t)=A \phi(z) \cos \alpha(\mathrm{x}-c t)
\end{aligned}
$$

Note that to the assumed $\Phi$ in eq 14 there corresponds in the liquid, and hence aiso at the plate/liquid interface, a sinusoidal wave of the type shown in eq 18 .

It was shown before that differential equation 21 and the boundary condition 23 are satisfied when

$$
\phi(z)=2 B_{1} \mathrm{e}^{a H} \cosh \alpha(H-z) .
$$

Substituting expressions 15 and 18 into condition 22 yields

$$
\left\{-2 A B_{1} \mathrm{e}^{\alpha H}[\sinh a(H-z)]_{\mathrm{z}=\mathrm{w}}+c w_{0}\right\} a \cos \alpha(x-c t)=0 .
$$

The above equation is satisfied when

$$
2 A B_{1}=\frac{c W_{0}}{e^{a H} \sinh a(H-w)} .
$$


Thus

$$
\Phi(x, z, t)=\frac{c w_{0} \cosh a(H-z)}{\sinh a(H-w)} \cos a(x-c t) \cong \frac{c w_{0} \cosh a(H-z)}{\sinh a H} \cos a(x-c t)
$$

since usually $w<<H$.

Substitution of $w(x, t)$ as given in eq 18 and of $\Phi(x, z, t)$ as shown above into differential equation 20 yields, noting that $w \ll H$,

$$
\left(D a^{4}-N_{a}^{2}-\rho_{\mathrm{p}} h a^{2} c^{2}+\rho_{\mathrm{f}} g-\rho_{\mathrm{f}} \frac{a c^{2} \cosh a H}{\sinh a H}\right) w_{0} \sin a(x-c t)=0 .
$$

The above equation is satisfied when

$$
a^{2}\left(a^{2}-\frac{N}{D}\right)+\frac{\rho_{\mathrm{f}} g}{D}-\frac{c_{\mathrm{pf}}^{2} \alpha^{2}}{D}\left[\rho_{\mathrm{p}} h+\frac{\rho_{\mathrm{f}}}{a} \operatorname{ctgh}(a H)\right]=0 .
$$

This is the dispersion relation for the stressed plate on a liquid base.

Thus,

$$
c_{\mathrm{pf}}^{2}=\frac{\left(D a^{2}-N\right)+\frac{\rho_{\mathrm{f}} \mathrm{g}}{a^{2}}}{\left[\rho_{\mathrm{p}} h+\frac{\rho_{\mathrm{f}}}{\operatorname{atg} h(a H)}\right]} .
$$

As expected, when the plate is absent $(D=N=h=0)$ eq 26 reduces to eq 16 , and when the liquid is absent $\left(\rho_{\mathrm{f}}=0\right)$ eq 26 reduces to eq 19.

\section{Denoting}

$$
\ell^{4}=\frac{D}{\rho_{\mathrm{f}} \mathrm{g}} \quad \mu=\frac{\rho_{\mathrm{p}} h}{\rho_{\mathrm{f}} \ell} \quad V_{\mathrm{pf}}^{2}=\frac{\mathrm{c}_{\mathrm{pf}}^{2}}{g l}
$$

eq 25 may be written as

$$
1+(a \ell)^{4}-\frac{N \ell^{2}}{D}(a \ell)^{2}-V_{\mathrm{pf}}^{2}\left[\mu(a \ell)^{2}+a \ell \operatorname{ctgh}\left(a \ell \frac{H}{l}\right)\right]=0
$$

and eq 26 as

$$
V_{\mathrm{pf}}^{2}=\frac{1+(a \ell)^{2}\left[(a \ell)^{2}-N \ell^{2} / D\right]}{(a \ell)} \frac{\operatorname{tgh}(a H)}{1+\mu a \ell \operatorname{tgh}(a H)} .
$$

A graphical presentation of eq 26 is shown in Figure 5 for $H=\infty, \rho_{\mathrm{f}}=1 \mathrm{~g} / \mathrm{cm}^{3}, \rho_{\mathrm{p}}=0.92 \mathrm{~g} / \mathrm{cm}^{3}, E=$ $50,000 \mathrm{~kg} / \mathrm{cm}^{2}, \nu=0.34$ and $h=30 \mathrm{~cm}$ (thus, $\ell=454 \mathrm{~cm}$ and $\mu=0.0609$ ). The situation is similar to that of a beam on a Winkler base discussed recently by $\operatorname{Kerr}(1972)$. Namely for a given $N<N_{\mathrm{cr}}$ a wave of the form 


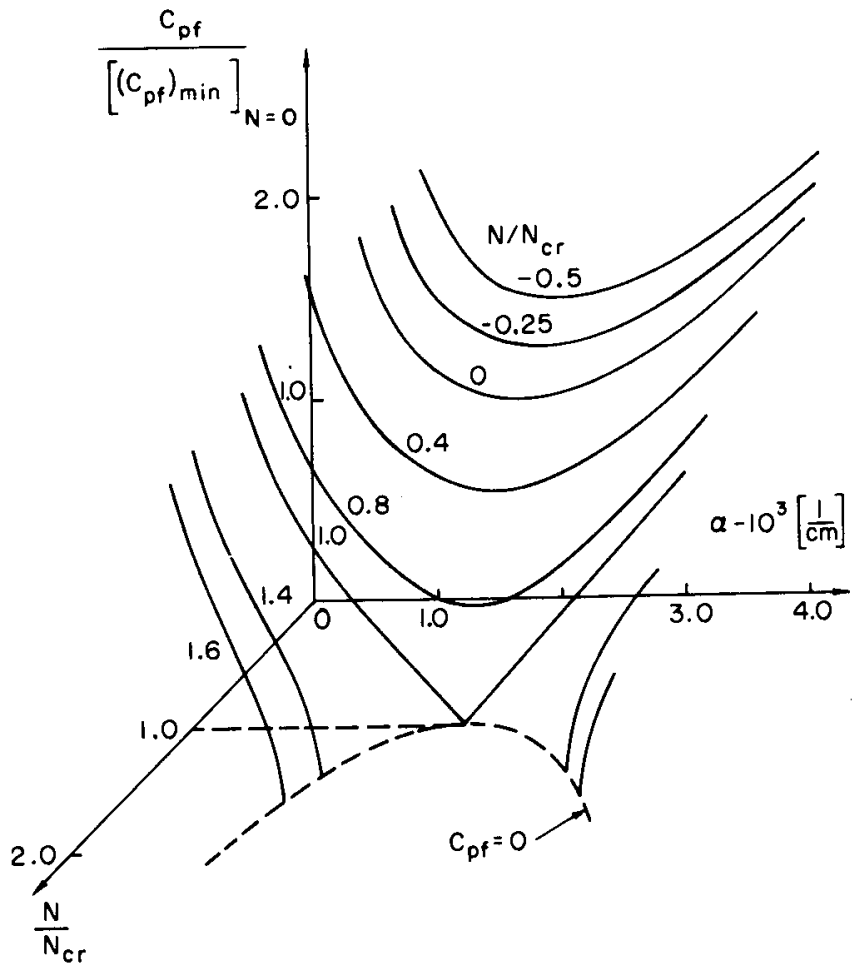

Figure 5.

18 cannot propagate for $0<c<\left(c_{\mathrm{pf}}\right)_{\min }$ and for each $c>\left(c_{\mathrm{pf}}\right)_{\min }$ two wave trains with different $\lambda$ may propagate.

The findings of Kerr (1972) as well as those of Kheishin (1967) suggest that $\left(c_{\mathrm{pf}}\right)_{\mathrm{min}}$ may be the critical velocity of a load $P$ which moves in the direction of the $x$-axis.

A necessary condition for locating $\left(c_{\mathrm{pf}}\right)_{\min }$ for a given $N$ is

$$
\frac{\partial c_{\mathrm{pf}}}{\partial a}=0
$$

It yields, using eq 26 , and then setting $N / a^{2} D=n$ to simplify the presentation of the final result,

$$
\left[1+(a \ell)^{4}(1-n)\right] a H-2 \mu(a \ell)\left[1-(a \ell)^{4}\right] \sinh ^{2}(a H)+1 / 2 \sinh (2 a H)\left[(a \ell)^{4}(3-n)-1\right]=0
$$

or, rewritten,

$$
\frac{1+(a \ell)^{4}(n-3)}{1+(a \ell)^{4}(1-n)}=\frac{2 a H}{\sinh (2 a H)}-\frac{\left[1-(a \ell)^{4}\right] 2 \mu(a \ell) \operatorname{tgh}(a H)}{1+(a \ell)^{4}(1-n)} .
$$

It is of interest to note that eq $26 \mathrm{a}$ and $27 \mathrm{a}$ for the case $N=0$ are the conditions for the determination of the critical velocity $v_{\mathrm{cr}}$ used by Nevel (1970).

The minimum value of $N$ is obtained from the condition

$$
\left(\frac{\partial N}{\partial a}\right)_{\mathrm{c}_{\mathrm{pf}}=0}=0
$$


It is found to take place at

$$
a=\sqrt[4]{\frac{\rho_{\mathrm{p}}}{D}}
$$

The corresponding

$$
\left(N_{\min }\right)_{\mathrm{c}_{\mathrm{pf}}=0}=2 \sqrt{\rho_{\mathrm{f}} g \bar{D}} .
$$

Thus, it is equal to the critical compression force $N_{\text {cr }}$ given in eq 9 .

\section{THE STRESSED FLOATING PLATE SUBJECTED TO A MOVING LOAD $P$}

It is assumed that the plate is subjected to a force $P$ which moves with a constant velocity $v_{0}$ as shown in Figure 6. The governing equations are:

$$
\begin{aligned}
& D \nabla_{\mathrm{x}, \mathrm{y}}^{4} w+N \nabla_{\mathrm{x}, \mathrm{y}}^{2} w+\rho_{\mathrm{p}} h \frac{\partial^{2} w}{\partial t^{2}}+\rho_{\mathrm{f}} \mathrm{gw}-\left.\rho_{\mathrm{f}} \frac{\partial \Phi}{\partial t}\right|_{\mathrm{z}=\mathrm{w}}=P \delta\left(\mathrm{x}-\mathrm{v}_{0} t\right) \delta(\mathrm{y}) \\
& \nabla_{\mathrm{x}, \mathrm{y}, \mathrm{z}}^{2} \Phi=0
\end{aligned}
$$

where

$$
\begin{aligned}
& \left.\frac{\partial \Phi}{\partial z}\right|_{\mathrm{z}=\mathrm{w}}=\frac{\partial w}{\partial t} \\
& \left.\frac{\partial \Phi}{\partial z}\right|_{\mathrm{z}=\mathrm{H}}=0
\end{aligned}
$$

and regularity conditions for $w$ and $\Phi$ as $x^{2}+y^{2} \rightarrow \infty$.

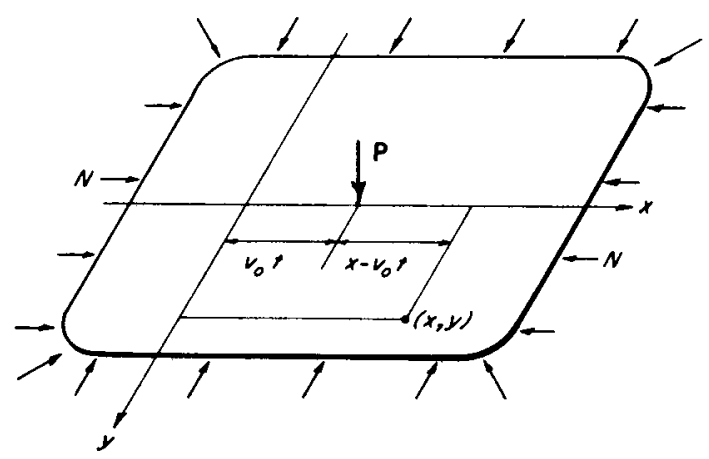

Figure 6. 
It is further assumed that after a time $t$ a steady state will exist. This assumption allows the time variable to be transformed out by means of the simple transformation

$$
\xi=\mathrm{x}-\mathrm{v}_{0} t \quad \eta=y \quad \zeta=z .
$$

The resulting formulation consists of differential equations

$$
\begin{aligned}
& D \nabla_{\xi, \eta}^{4} w+N \nabla_{\xi, \eta}^{2} w+\rho_{\mathrm{p}} h \mathrm{v}_{0}^{2} \frac{\partial^{2} w}{\partial \xi^{2}}+\rho_{\mathrm{f}} \boldsymbol{g w}+\left.\rho_{\mathrm{f}} \mathrm{v}_{0} \frac{\partial \Phi}{\partial \xi}\right|_{\zeta=\mathrm{w}}=P \delta(\zeta) \delta(\eta) \\
& \nabla_{\xi, \eta, \zeta}^{2} \Phi=0
\end{aligned}
$$

with the boundary conditions

$$
\begin{aligned}
& \left.\frac{\partial \Phi}{\partial \zeta}\right|_{\zeta=\mathrm{w}}=-\mathrm{v}_{0} \frac{\partial w}{\partial \xi} \\
& \left.\frac{\partial \Phi}{\partial \zeta}\right|_{\zeta=\mathrm{H}}=0
\end{aligned}
$$

and the regularity conditions for $w$ and $\Phi$ as $x^{2}+y^{2} \rightarrow \infty$

$$
\lim _{\xi, \eta \rightarrow \pm \infty}\left(w, \Phi, \frac{\partial w}{\partial \xi}, \frac{\partial w}{\partial \eta}, \ldots, \frac{\partial \Phi}{\partial \xi}, \frac{\partial \Phi}{\partial \eta}, \ldots\right) \rightarrow 0 .
$$

The resulting formulation differs from the one used by Kheishin $(1963,1967)$ and Nevel (1970) in that it contains the term $N \nabla_{\xi \eta}^{2} w$ in the first equation. To solve the posed problem we proceed according to Kheishin (1967) and Nevel (1970).

We introduce the double Fourier transforms

$$
\begin{aligned}
& \bar{w}(\alpha, \beta)=\int_{-\infty}^{\infty} \int_{-\infty}^{\infty} w(\xi, \eta) \mathrm{e}^{-i(\alpha \xi+\beta \eta)} \mathrm{d} \xi \mathrm{d} \eta \\
& \bar{\Phi}(\alpha, \beta, \zeta)=\int_{-\infty}^{\infty} \int_{-\infty}^{\infty} \Phi(\xi, \eta, \zeta) \mathrm{e}^{-i(a \xi+\beta \eta)} \mathrm{d} \xi d \eta
\end{aligned}
$$

Multiplying eq 33 by $\mathrm{e}^{-i(\alpha \xi+\beta \eta)}$ and integrating it as indicated in eq 38 we obtain

$$
\int_{-\infty}^{\infty} \int_{-\infty}^{\infty}\left(\frac{\partial^{2} \Phi}{\partial \xi^{2}}+\frac{\partial^{2} \Phi}{\partial \eta^{2}}+\frac{\partial^{2} \Phi}{\partial \xi^{2}}\right) \mathrm{e}^{-i(\alpha \xi+\beta \eta)} \mathrm{d} \xi \mathrm{d} \eta=0
$$

Using integration by parts, and noting regularity conditions 36 and definition 38 , the above equation becomes

$$
\frac{\partial^{2} \bar{\Phi}}{\partial \zeta^{2}}-\gamma^{2} \bar{\Phi}=0 \quad \text { for } 0 \leqq \zeta<\infty
$$


where

$$
\gamma^{2}=a^{2}+\beta^{2}
$$

The same transformation performed on the boundary conditions 34 and 35 yields

$$
\begin{aligned}
& \left.\frac{\partial \Phi}{\partial \zeta}\right|_{\zeta=\mathrm{w}}=-i a \mathrm{v}_{0} \overline{\mathcal{w}} \\
& \left.\frac{\partial \bar{\Phi}}{\partial \zeta}\right|_{\zeta=\mathrm{H}}=0 .
\end{aligned}
$$

The general solution of eq 39 is

$$
\bar{\Phi}=A_{1} \mathrm{e}^{-\gamma \zeta}+A_{2} \mathrm{e}^{+\gamma \zeta}
$$

Noting the boundary conditions in eq 41 and $42, \bar{\Phi}$ becomes

$$
\bar{\Phi}(\alpha, \beta, \zeta)=\frac{i a \mathrm{v}_{0} \bar{W}}{\gamma} \frac{\cosh \gamma(H-\zeta)}{\sinh \gamma(H-w)} .
$$

For $w<<$, the above equation simplifies to

$$
\bar{\Phi}(\alpha, \beta, \zeta) \cong\left[\frac{i a v_{0}}{\gamma} \frac{\cosh \gamma(H-\zeta)}{\sin \gamma H}\right] \bar{W}(\alpha, \beta) .
$$

The same transformation performed on differential equation 32 yields

$$
\left[D\left(\alpha^{4}+2 \alpha^{2} \beta^{2}+\beta^{4}\right)-N\left(\alpha^{2}+\beta^{2}\right)-\rho_{\mathrm{p}} h \mathrm{v}_{0}^{2} \alpha^{2}+\rho_{\mathrm{f}} g\right] \overline{\mathrm{w}}+\left.i \rho_{\mathrm{f}} \mathrm{v}_{0} \alpha \bar{\Phi}\right|_{\zeta=\mathrm{w}}=P .
$$

Since according to eq 45 , for $w<H$,

$$
\left.\bar{\Phi}\right|_{\zeta=\mathrm{w}} \cong \frac{i a \mathrm{v}_{0}}{\gamma \operatorname{tgh} \gamma H} \bar{W}
$$

eq 46 becomes, noting eq 40 ,

$$
\left[D \gamma^{4}-N \gamma^{2}+\rho_{\mathrm{f}} g-v_{0}^{2}\left(\rho_{\mathrm{p}} h \alpha^{2}+\frac{\rho_{\mathrm{f}} \alpha^{2}}{\gamma \operatorname{tgh} \gamma H}\right)\right] \bar{w}=P .
$$

Denoting

$$
\ell^{4}=\frac{D}{\rho_{\mathrm{f}} \boldsymbol{g}} \quad \mu=\frac{\rho_{\mathrm{p}} h}{\rho_{\mathrm{f}} \ell} \quad V_{0}^{2}=\frac{\mathrm{v}_{0}^{2}}{\mathrm{~g} \ell}
$$

eq 47 may be rewritten as follows: 


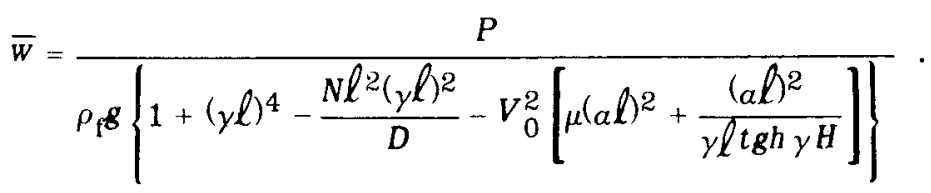

Inverting $\bar{w}$, it follows that

$$
w(\xi, \eta)=\frac{P}{4 \pi^{2} \rho_{\mathrm{f}} \xi} \int_{-\infty}^{\infty} \int_{-\infty}^{\infty} \frac{\mathrm{e}^{i(\alpha \xi+\beta \eta)}}{\mathrm{a}-\mathrm{b} a^{2} / \gamma^{2}} d a d \beta
$$

where

$$
\begin{aligned}
& \mathrm{a}=1+(y \ell)^{4}-\frac{N \ell^{2}}{D}(y \ell)^{2} \\
& \mathrm{~b}=V_{0}^{2}\left[\mu(y \ell)^{2}+\gamma \ell \operatorname{ctgh}\left(\gamma \ell \frac{H}{\ell}\right)\right] .
\end{aligned}
$$

It may be shown, as done by Nevel $(1970)$, that $w(\xi, \eta) \rightarrow \infty$ when

$$
\mathrm{a}=\mathrm{b}
$$

and

$$
\frac{\partial \mathrm{a}}{\partial \gamma}=\frac{\partial \mathrm{b}}{\partial \gamma}
$$

Nevel used these two conditions to determine the critical velocity $v_{0}$.

It should be noted that these two conditions are identical to eq $25 \mathrm{a}$ and eq $27 \mathrm{a}$, when the variable $(\gamma \ell)$ is replaced by $(a \ell)$ and the parameter $V_{0}$ by $V_{\mathrm{pf}}$. Since the variables $(\gamma \ell)$ or $(a \ell)$, determined from eq 52 or $27 \mathrm{a}$, are identical, it follows that eq 52 for the determination of $V_{0}$ and eq $25 \mathrm{a}$ for the determination of $V_{\mathrm{pf}}$ are identical. Thus, as expected, the critical velocity $\mathrm{v}_{0}$ is the same as $\left(c_{\mathrm{pf}}\right)_{\min }$. Hence, the dependence of the critical velocities $v_{\mathrm{cr}}$ upon the axial force field $N$ is as shown in Figure 5 for $\left(c_{\mathrm{pf}}\right)_{\min }$ and is shown in Figure 8 for $h=30 \mathrm{~cm}$ and $90 \mathrm{~cm}$. The dependence of $\mathrm{v}_{\mathrm{cr}}$ upon $N$ and $h$ was obtained by numerically evaluating eq 26 and 27 for $H=\infty$, noting that $\left(c_{\mathrm{pf}}\right)_{\min }=\mathrm{v}_{\mathrm{cr}}$. The obtained results are shown in Figure 9.

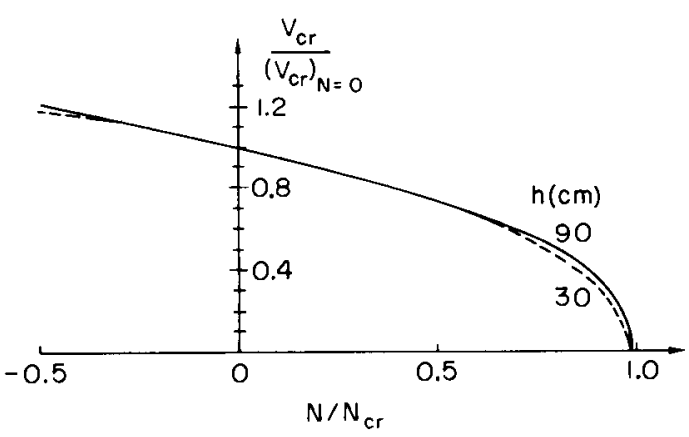

Figure 8.

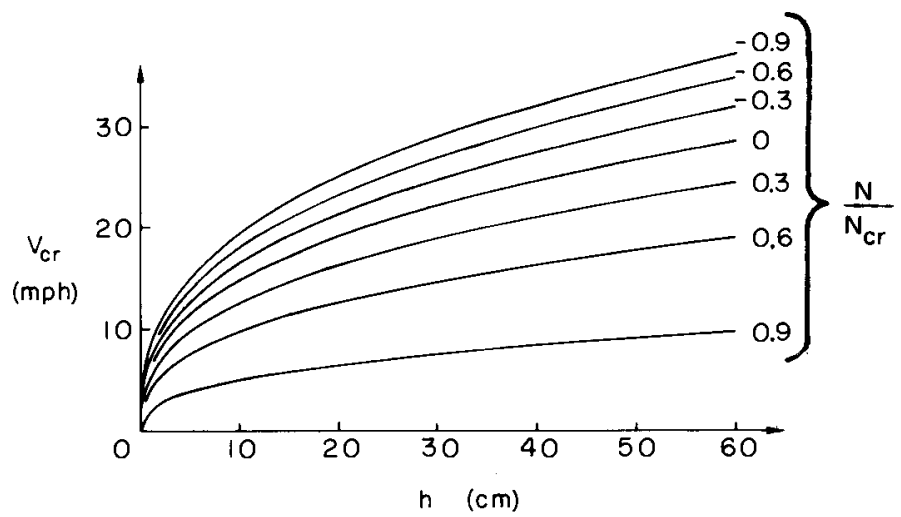

Figure 9. 


\section{CONCLUSION}

The effect of a uniform in-plane force field in a floating plate upon the critical velocities of a moving load has been studied. For an increasing compression force field the critical velocity decreases, approaching the value zero as $N \rightarrow N_{\mathrm{cr}}$; for an increasing tension force field $\mathrm{v}_{\mathrm{cr}}$ increases.

\section{LITER ATURE CITED}

Assur, A. (1961) Traffic over frozen or crusted surfaces. Proceedings of the First International Conference on the Mechanics of Soil-Vehicle Systems, Edizioni Minerva, Torino, Italy, Tecnica.

Kerr, A.D. (1972) The continuously supported rail subjected to an axial force and a moving load. International Journal of Mechanical Sciences, vol. 14.

Kheishin, D.E. (1963) Moving load on an elastic plate which floats on the surface of an ideal liquid (in Russian). Izvestia AN SSSR OTN, Mekhanika i Mashinostroenie.

Kheishin, D.E. (1967) Dynamics of the ice cover (in Russian). Gidrometeorologicheskoie Izdatelstvo, Leningrad.

Lamb, H. (1945) Hydrodynamics. Dover.

Nevel, D.E. (1970) Moving loads on a floating ice sheet. U.S. Army Cold Regions Research and Engineering Laboratory (USA CRREL) Research Report 261. 